\title{
Remarkable prolonged progression-free survival time after therapy with trifluridine/ tipiracil. A case report of 57-year-old woman with metastatic colorectal cancer
}

Marlena Orlikowska

Oncology and Chemotherapy Outpatient Clinic, Hospital in Starogard Gdański

Correspondence: Oncology and Chemotherapy Outpatient Clinic, Hospital in Starogard Gdański 83-200 Starogard Gdański, ul. dra Józefa Balewskiego 1 phone: (58) 7749611

Received:

12.03.2020

Accepted:

25.03.2020

DOI: 10.24292/01.OR.120250320

Copyright $\odot$ Medical Education. All rights reserved.

\section{ABSTRACT}

Colorectal cancer is the second leading cause of cancer-related death worldwide. Trifluridine/tipiracil is a novel orally administered antineoplasmatic thymidine-based nucleoside analog which represents an approved option for the treatment of advanced metastatic colorectal cancer in patients who are refractory, or are not considered candidates for, currently available therapies. In this report, we present a case of chemotherapy using trifluridine/tipiracil confirming the effectiveness of this drug primarily in the term of prolongation of progression free survival.

Key words: colorectal cancer, chemotherapy, trifluridine/tipiracil 


\section{INTRODUCTION}

Colorectal cancer (CRC) is the second leading cause of cancer-related death worldwide [1]. Rapid increases in both CRC incidence and mortality are now observed in many medium-to-high human development index countries particularly in Eastern Europe, Asia and South America [2]. According to Wilkes [3], approximately $15-25 \%$ of newly diagnosed patients demonstrate criteria for metastatic CRC (mCRC) and around $50-60 \%$ of CRC patients will finally develop metastases which definitely worsen the prognosis in the course of the disease.

Trifluridine/tipiracil (FTD/TPI) is a novel orally administered antineoplasmatic thymidine-based nucleoside analog which represents an approved option for the treatment of advanced mCRC in patients who are refractory, or are not considered candidates for currently available therapies [4].

Trifluridine (FTD) which inhibits thymidylate synthase (crucial enzyme of DNA synthesis) is the active cytotoxic component of the FTD/TPI, while TPI improves the bioavailability of the latter. Additional antineoplasmatic activity of FTD/TPI which distinguishes it from traditional fluoropyrimidines results from trifluridine incorporation into DNA which finally leads to double-helical DNA damage [5].

The efficacy of FTD/TPI in the treatment of $\mathrm{mCRC}$ has been confirmed in the randomized phase III clinical trial RECOURSE. Therapy with FTD/TPI, significantly prolonged median overall survival (OS) compared to placebo (7.1 vs. 5.3 months; hazard ratio [HR] $0.68 ; p<0.0001$ ). In addition FTD/TPI significantly prolonged median progression free survival (PFS): 2.0 vs. 1.7 months; $H R=0.48$ $\mathrm{p}<0.0001$, improved disease control rate and prolonged median time to deterioration of performance status compared to placebo [6].

In this report, we present a case of $\mathrm{mCRC}$ chemotherapy using FTD/TPI confirming the effectiveness of this drug primarily in the term of prolongation of PFS.

\section{CASE REPORT}

In July 2015, a 57-year-old patient underwent surgery for sigmoid cancer with the Hartmann method due to obstruction. During the procedure, the sigmoid tumor was removed with surgical margin, and the specimens were taken from the ruptured right ovarian cyst. The histopathological findings revealed: tumor - adenocarcinoma invasivum intestini crassi G1, whereas in the ovarian slice - cystadenocarcinoma endometrioides ovarii was found.

The staging was assessed at PT2N1Mx. As the synchronous cancer of the ovary was suspected, gynecological cytoreduction was performed in September 2015. The histopathological findings showed: tissue of intestinal adenocarcinoma in the right ovary, in other postoperative material with no cancer cells infiltration. The staging was changed to PT2N1M1. Subsequently the patient was qualified for FOLFIRI chemotherapy (irinotecan, fluorouracil). The patient received 12 infusions scheduled for the period November 2015 - April 2016.

In November 2016, it was decided to perform a PET examination due to the increase in the value of the CEA marker over time, whereas there was no disease progression in computed tomography. PET revealed liver metastasis $18 \times 15 \mathrm{~mm}$ SUV - 13.5 and intraperitoneal cancer implants up to $7 \mathrm{~mm}$ with SUV 5.5. It was decided to include II line chemotherapy with FOLFOX-4 (oxaliplatin, fluorouracil). The treatment was complicated by hematological toxicity (CTC grade III neutropenia and CTC grade III thrombocytopenia). Prednisone induction was used with good results. After six courses in April 2017 PET examination was performed again (with possibility to perform metastasectomy), in which metabolic complete remission of neoplastic lesions was found. After agreement with the patient, the treatment was postponed and the patient was observed.

In July 2017, an attempt was made to reconstruct the continuity of the gastrointestinal tract (in CT scan no tumor lesions were found), but intraperitoneal implants were found intraoperatively, which was confirmed by histopathological examination. Therefore, stoma removal surgery was abandoned.

After a 5-month break in treatment, the CEA marker increased again to $98 \mathrm{ng} / \mathrm{mL}$ (August 2017), CT revealed enlarged lymph nodes of the presacral area up to $13 \mathrm{~mm}$ and small nodular lesions in the lungs up to $5 \mathrm{~mm}$. FOLFOX-4 was administered again (from September 2017). Because of the above mentioned toxicity of the treatment, stimulation with prednisone and short-acting GCSF (granulocyte growth factor) in secondary prevention was included. After administration of five treatment courses, anaphylaxis to oxaliplatin occurred, so treatment with the LF4 (5-fluorouracil + leucovorin) schedule was continued until March 2018. CT scan of the thorax, abdomen and pelvis after month as the treatment ended, showed complete regression of nodal lesions and stabilization in the size of lumps in the lungs. In the liver there were no metastatic lesions. The CEA marker at the time was $27 \mathrm{ng} / \mathrm{mL}$. 
Considering peritoneal metastases, which are hardly visible in imaging examinations, it was decided to refer the patient for consultation to the PIPAC performing center (Pressurized Intraperitoneal Aerosol Chemotherapy). However, the patient was not qualified for this procedure. At the same time, histopathological specimens were sent for the examination for RAS/BRAF mutation, which were confirmed.

The patient was observed, since August 2018 to October 2018 the CEA marker (sensitive in our patient) increased from $94 \mathrm{ng} / \mathrm{mL}$ to $200 \mathrm{ng} / \mathrm{mL}$. It was decided to include FTD/TPI (fig. 1). She received a dose of $2 \times 60 \mathrm{mg}$ on days $1^{\text {st }}-5^{\text {th }}$ and $8^{\text {th }}-12^{\text {th }}$ on a 28-day cycle.

FIGURE 1.

CT scan of the chest and peritoneum - 3.10.2018 r.

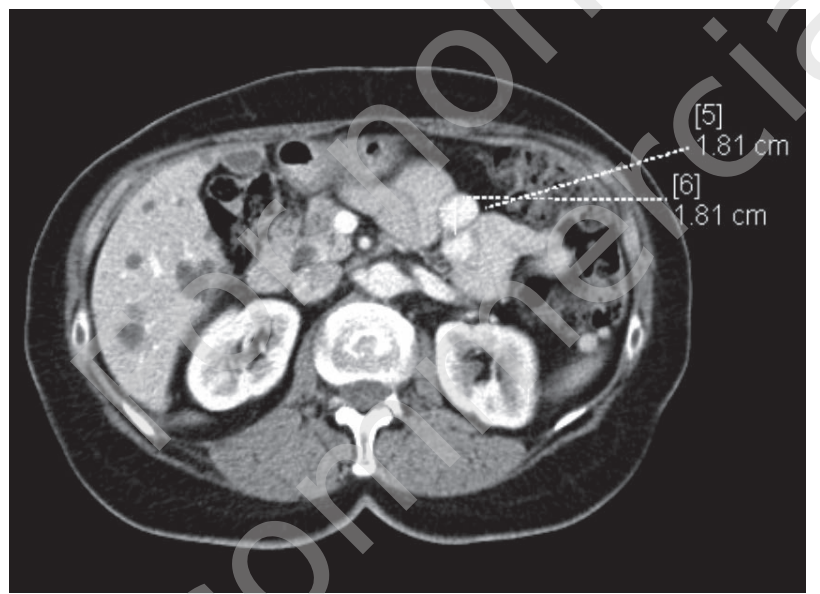

Already after the first course of FTD/TPI therapy, neutropenia in CTC grade III and a decrease in the CEA marker from 200 to $132 \mathrm{ng} / \mathrm{mL}$ were observed. The patient reported weakness in CTC grade I. The second course was given with a week delay after obtaining an increase in neutrophilia. It was recommended to include prednisone $20 \mathrm{mg}, 3$ days before the next chemotherapy course in order to improve bone marrow function parameters. However, after the second course, in the time when the III cycle of treatment was supposed to be started, CTC grade III neutropenia was again observed - the GCSF was administered.

After 3 months of treatment, computed tomography of the chest and abdominal cavity with pelvis was performed, where regression of the peritoneal implants was found by about $40 \%$ on the RECIST scale (fig. 2). Therefore, treatment was continued, during which there was an increase in the number of side effects. After the forth course, the patient lost $4 \mathrm{~kg}$ (from 75 to $71 \mathrm{~kg}$ ) as an effect of worsening appetite, taste disturbances and diarrhea in CTC grade I. A small alopecia also occurred. The CEA marker after the forth course remained at $159 \mathrm{ng} / \mathrm{mL}$. In the following months, the patient complained of increasing fatigue, which interfered with her daily duties, and the patient's general condition was assessed for PS-1. In the next tomographic examination performed on April $2^{\text {nd }}, 2019$ (before the sixth course) stabilization of lesions compared to the previous study was found. The CEA marker at that time was $181 \mathrm{ng} / \mathrm{mL}$. Eighth course of treatment was given, which was tolerated by the patient badly and caused vomiting, fatigue and weakness in grade PS-2, the patient lost $3 \mathrm{~kg}$ (weight in July $2019-68 \mathrm{~kg}$ ), and the CEA marker increased to $230 \mathrm{ng} / \mathrm{mL}$.

FIGURE 2.

CT scan of the chest and peritoneum - 3.01.2019 r.

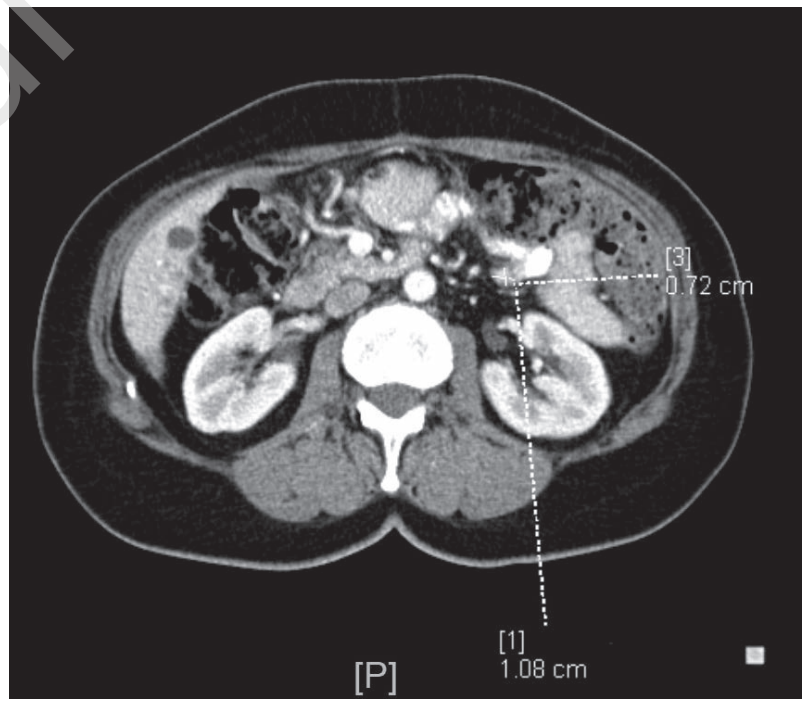

The FTD/TPI intake was withheld. An urgent tomographic examination was commissioned, which was carried out on August $5^{\text {th }}$, 2019 (a month after discontinuation of FTD/TPI), where progression of metastatic changes was observed, mainly in the area of the abdominal wall. During this time, CEA increased from 230 to $1056 \mathrm{ng} / \mathrm{mL}$ (within a month) and neuropathic abdominal pain appeared. Analgesic treatment was set up and it was proposed to include another line of chemotherapy - FOLFIRI. The patient is currently continuing treatment with the above schedule, she obtained significant regression of the CEA marker after just one month of treatment and reduced abdominal pain.

\section{DISCUSSION}

Fluoropyrimidines are still mainstay of the chemotherapy schedules for mCRC patients, both as initial therapy and as important option in subsequent therapy, alone or in combination with oth- 
er drugs (including biological targeted agents such as anti-vascular endothelial growth factor - VEGF, monoclonal antibodies - e.g. bevacizumab and anti-epidermal growth factor receptor - EGFR - monoclonal antibodies - e.g. cetuximab). Treatment is administered until the disease progress, recurs, or the toxicity of therapy is intolerable by the patient [7].

According to the guidelines of European Society for Medical Oncology (ESMO) and the statement of the European Medicines Agency (EMA) trifluridine/tipiracil is the oral agent approved in the palliative setting for the treatment of $\mathrm{mCRC}$ in patients who are refractory, or are not considered candidates for currently available therapies $[4,8]$. The combined properties of FTD/TPI provide a novel therapeutic option for $\mathrm{mCRC}$ patients which are refractory, among others, to traditional fluoropyrimidines (e.g. 5-fluorouracil).

The basis for introducing the FTD/TPI to the current guidelines were the results of the RECOURSE clinical trial published in 2015. In the RECOURSE trial, the addition of FTD/TPI to best supportive therapy resulted in a modest, but clinically meaningful and statistically significant improvement in the median OS. Therapy with FTD/TPI was also associated with a significantly longer PFS and a higher disease control than placebo. The most common adverse events were haematological and gastrointestinal and were observed more often during the first dosing cycle of FTD/ TPI [6].

Our case concerned a "typical" patient with $\mathrm{mCRC}$ qualified for the RECOURSE study, i.e. not eligible or refractory for currently available therapies. Despite the significant advancement of the neoplastic process, the therapy with FTD/TPI was well tolerated for a long time and the side effects were controlled without major clinical problems. It should be noted that the progression free survival time ( 8 months) observed in our patient was substantially longer than observed in the RECOURSE trial (about 2 months).

\section{CONCLUSION}

Our observation confirms that FTD/TPI is an important, novel therapeutic option in patients with advanced metastatic colorectal cancer, which, above all, can prolong the time of progression of the proliferative process.

\section{References}

1. International Agency for Research on Cancer: GLOBOCAN 2018. http://gco.iarc.fr/today/data/factsheets/cancers/10_8_9-Colorectum-fact-sheet. pdf.

2. Center MM, Jemal A, Smith RA et al. Worldwide variations in colorectal cancer. CA Cancer J Clin. 2009; 59: 366-78.

3. Wilkes GM. Metastatic colorectal cancer: management challenges and opportunities. Oncology. 2011;25(7):32-44.

4. Lenz HJ, Stintzing S, Loupakis F. TAS-102, a novel antitumor agent: a review of the mechanism of action. Cancer Treatment Rev. 2015; 41: 777-83.

5. Burness CB, Duggan ST. Trifluridine/tipiracil: a review in metastatic colorectal cancer. Drugs. 2016; 76: 1393-402.

6. Mayer R, Van Cutsem E, Falcone A et al. RECOURSE Study Group Randomized trial of TAS-102 for refractory metastatic colorectal cancer. N Engl J Med. 2015; 372: 1909-19.

7. Van Cutsem E, Cervantes A, Adam R et al. ESMO consensus guidelines for the management of patients with metastatic colorectal cancer. Ann Oncol. 2016; 27 (8): 1386-422.

8. European Medicines Agency. European public assessment report (EPAR) for Lonsurf. 2016. http://www.ema.europa.eu. 